\title{
Modeling the Stress Dependence of Magnetic Hysteresis Based on Stoner-Wohlfarth Theory
}

\author{
Weijie Xu, Nana Duan, Shuhong Wang \\ State Key Laboratory of Electrical Insulation and Power \\ Equipment, School of Electrical Engineering, \\ Xi'an Jiaotong University, \\ Xi'an 710049, China \\ shwang@mail.xjtu.edu.cn
}

\author{
Youguang Guo, Jian Guo Zhu \\ School of Electrical, Mechanical and Mechatronic Systems, \\ University of Technology Sydney, \\ NSW 2007, Australia \\ jianguo.zhu@uts.edu.au
}

\begin{abstract}
This paper presents an improved approach for simulating magnetic hysteresis, which takes into account the effect of applied stress, based on an extended Stoner-Wohlfarth (S-W) model. Meanwhile, the S-W asteroid rotates and shrinks, and the stable direction of magnetization of the particle can be calculated from the new energy minimum conditions. This developed model is applied to analyze the magnetic hysteresis phenomenon of a soft magnetic composite (SMC) material under different compaction process, and the results of simulations are in good quantitative agreement with experimental data.
\end{abstract}

Keywords- Stoner-Wohlfarth Model; Stress effect; Hysteresis Model; Soft Magnetic Composite Materials

\section{INTRODUCTION}

The stress effect on the magnetic material is a significant contributing factor during the magnetization processes [1]. In order to understand thoroughly the behavior of magnetization processes in magnetic materials at different applied stresses, a stress-dependent hysteresis model is necessary. Model of stress-dependent magnetic hysteresis has been under investigation for many years and various approaches have been proposed [2][3]. Among the previous works, many features in the model cannot be reconciled with the physical mechanism of the magnetic material.

In this paper, the stress effect can be taken into account by adding the magnetoelastic energy into the traditional S-W model, and after mathematical transformation, the expression of the total energy can also be formulated as an equivalent S-W model with a rotation and scaling on the S-W asteroid. Finally, the numerical simulation results of the magnetization of a soft magnetic composite (SMC) material are compared with the measured data.

\section{MODEL DESCRIPTION}

In this extended $\mathrm{S}-\mathrm{W}$ model, the magnetization direction is determined from the various energy contributions of the material anisotropy, applied field and the mechanical stress, as illustrated in Fig. 1. Then, the energy of a single S-W particle can be rewritten as:

$$
E=K \sin ^{2} \theta_{M}-\mu_{0} M_{S} H \cos \left(\theta_{M}-\theta_{H}\right)-\frac{3}{2} \lambda \sigma \sin 2\left(\theta_{M}-\theta_{\sigma}\right)
$$

where $\theta_{H}, \theta_{M}$ and $\theta_{\sigma}$ are the angles of the applied field, the resultant magnetization, and stress, respectively, with respect to the particle's anisotropic (easy) axis, $\lambda$ is the magnetostriction constant, and $\sigma$ is the applied mechanical stress. The first term represents the anisotropic energy, the second the interaction energy associated with the external magnetic field and the particle magnetization, and the third the magnetoelastic energy.

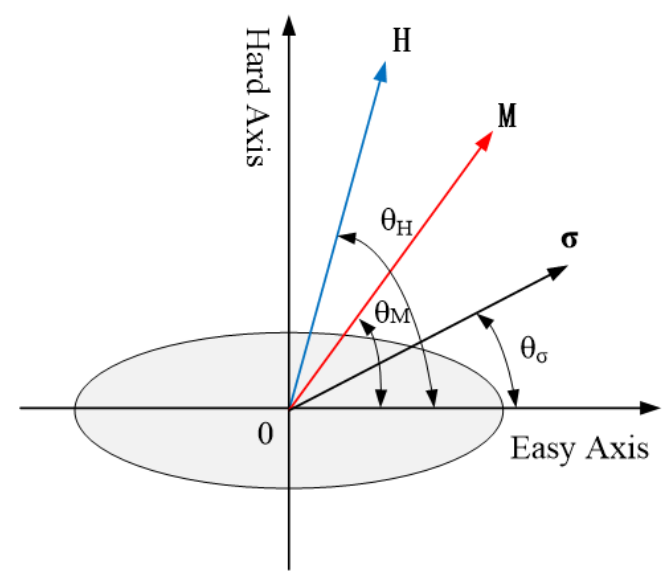

Figure.1 Orientation of a uniaxial S-W particle with a horizontal anisotropy axis

Based on this developed equation, a new direction of minimum energy can be obtained. Thus, on the basis of mathematic deduction, an equivalent energy model is proposed to include the effect of stress, giving

$$
E=K_{e f f} \sin ^{2}\left(\theta_{M}-\theta_{\text {rot }}\right)-\mu_{0} M_{S} H \cos \left(\theta_{M}-\theta_{H}\right)
$$

where $\theta_{\text {rot }}$, an additional rotation caused by the applied stress, is defined as follows:

$$
\theta_{r o t}=\frac{1}{2} \arccos \left[\frac{K}{K_{e f f}}\left(1+h_{\sigma} \cos 2 \theta_{\sigma}\right)\right]
$$

in which the reduced stress quantity $h_{\sigma}=3 \lambda \sigma / K$, and the equivalent anisotropy constant $K_{\text {eff }}$ is now:

$$
K_{e f f}=K \sqrt{1+h_{\sigma}^{2}+2 h_{\sigma} \cos 2 \theta_{\sigma}}
$$


The stable direction of magnetization of the particle can be calculated from the energy minimum conditions. With the employment of normalized field $h_{\text {eff }}$, the energy minimum condition can be expressed as follows.

$$
\begin{aligned}
& \frac{\partial E}{\partial \theta_{M}}=\frac{1}{2} \sin 2\left(\theta_{M}-\theta_{r o t}\right)-h_{e f f} \sin \left(\theta_{M}-\theta_{H}\right)=0 \\
& \frac{\partial^{2} E}{\partial \theta_{M}^{2}}=\cos 2\left(\theta_{M}-\theta_{r o t}\right)-h_{e f f} \cos \left(\theta_{M}-\theta_{H}\right) \geq 0
\end{aligned}
$$

with the normalized field,

$$
h_{\text {eff }}=\frac{\mu_{0} M_{S} H}{2 K_{\text {eff }}}
$$

Then, a rotation and scaling will exert on the classical S-W astroid, as shown in Fig.2.

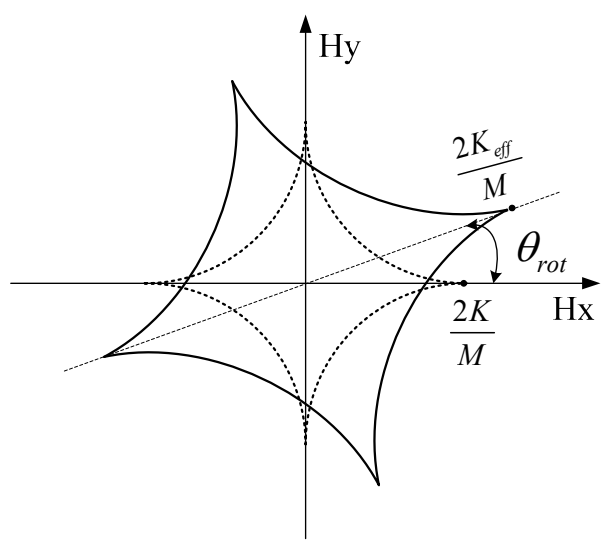

Figure.2 The original S-W asteroid (dashed) and the extended S-W asteroid (solid).

For a given applied field $\boldsymbol{H}$, the component of the total magnetization parallel to the applied field $\boldsymbol{M}$ can then be calculated by the following integral

$$
M=\int_{-\frac{\pi}{2}}^{\frac{\pi}{2}} \iint_{\alpha \geq \beta} \rho(\alpha, \beta, \theta) \gamma_{\alpha, \beta, \theta}(\mathrm{H}, \sigma) \mathrm{d} \alpha \mathrm{d} \beta \mathrm{d} \theta
$$

where $\rho(\alpha, \beta, \theta)$ is the distribution function of the $\mathrm{S}-\mathrm{W}$ particles, and $\gamma_{\alpha \beta \theta}(H, \sigma)$ the output magnetization of the extended S-W operator under different applied field and stress, In addition, $\gamma_{\alpha \beta \theta}(H, \sigma)$ can also be determined directly, with the employment of the improved asteroid, as shown in Fig.2.

All the parameters of distribution function can be determined with the comparison of the key points (saturation, residual magnetism, and coercive force) on the hysteresis loop.

\section{SIMULATION AND VALIDATION}

The stress-dependent S-W model was validated against measurements made on soft magnetic composite (SMC) material by a $3 \mathrm{D}$ magnetic property tester [4]. The sample of SMC material in measurement was compacted with a highpressure $(800 \mathrm{MPa})$ in mold along the coordinate axis of $y$ direction.
For better readability, considering the symmetry of the hysteresis loop, only the upper half of the hysteresis major loops, under stress on the $\mathrm{y}$ axis and no stress on the $\mathrm{x}$ axis, are illustrated in Fig.3.

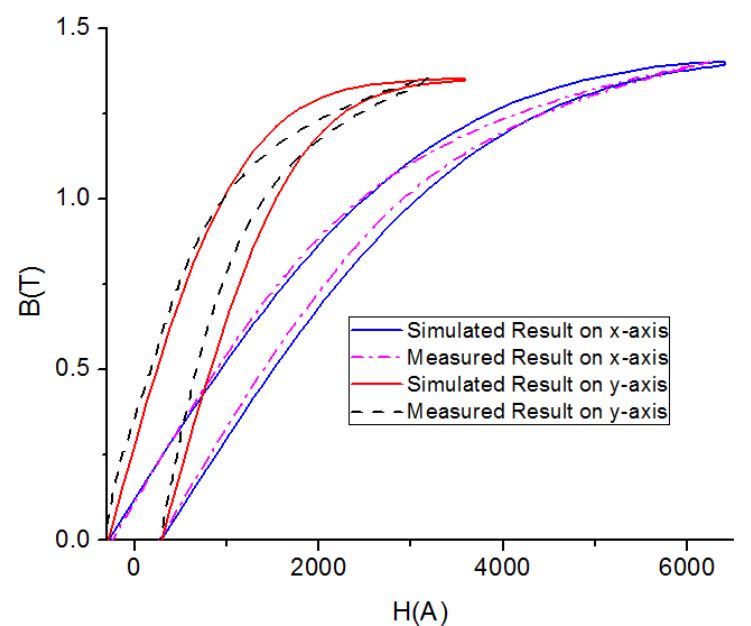

Figure. 3 Comparison of measured and simulated major B-H loops. The stress is applied along the y axis.

It is evident from the comparison that this stress-dependent $\mathrm{S}-\mathrm{W}$ model is in good agreement with the measurements.

\section{CONCLUSIONS}

In this work, a stress-dependent hysteresis model based on an extension of S-W theory was developed. The stress effects were incorporated into the existing $\mathrm{S}-\mathrm{W}$ model by considering the magnetoelastic energy, which is caused by the stress effect, into the total energy contributions. Then, the S-W asteroid will change due to the modification, and the stable direction of magnetization of the particle can be calculated from the new energy minimum conditions. The theory agrees well with the experimental results of an SMC sample measured by a 3D magnetic property tester.

\section{ACKNOWLEDGMENT}

The authors gratefully acknowledge the support for this research from the China Scholarship Council (CSC) (201306280073).

\section{REFERENCES}

[1] D. C. Jiles and M. K. Devine, "Recent developments in modeling of the stress derivative of magnetization in ferromagnetic materials," Journal of Applied Physics, vol. 76, pp. 7015-7017, 1994.

[2] A. Sipeky and A. Ivanyi, "Magnetic hysteresis under applied stress," Physica B: Condensed Matter, vol. 372, pp. 177-180, 2006.

[3] O. Perevertov, J. Thielsc,h and R. Schäfer, "Effect of applied tensile stress on the hysteresis curve and magnetic domain structure of grainoriented transverse $\mathrm{Fe}-3 \% \mathrm{Si}$ steel," Journal of magnetism and magnetic materials, vol. 385, pp. 358-367, 2015.

[4] Y. Guo, J. Zhu, Z. Lin, et al., "3D vector magnetic properties of soft magnetic composite material," Journal of magnetism and magnetic materials, vol. 302, pp. 511-516, 2006. 